\title{
A CHARACTERIZATION OF INACCESSIBLE CARDINALS
}

\author{
by G. B. PRESTON
}

(Received 20 January, 1961 and in revised form 24 August, 1961)

A cardinal number which is too large to be reached by some process is generally said to be inaccessible by that process. Many kinds of inaccessible cardinals have been discussed and for a general survey the book of $\mathrm{H}$. Bachmann [1, Chapter 7] may be consulted. We consider here two inaccessibility properties. We shall denote the cardinal of a set $X$ by $|X|$. The first inaccessibility property will be called regularity: the cardinal $|X|$ will be said to be regular if there does not exist a disjoint cover $\left\{X_{i}: i \in I\right\}$ of $X$ such that

(i) $\left|X_{i}\right|<|X|$, for each $i$ in $I$, and

(ii) $|I|<|X|$.

The second property is that of power inaccessibility. The cardinal $|X|$ is said to be power inaccessible if for any sets $Y$ and $Z$ such that $|Y|$ and $|Z|$ are less than $|X|$ we have $\left|Y^{Z}\right|<|X|$. Here $Y^{Z}$ denotes the set of all mappings of $Z$ into $Y$. A cardinal which is both power inaccessibie and regular will be said, simply, to be inaccessible. The paper of A. Tarski [2] is devoted to the discussion of cardinals which are inaccessible in this sense. Tarski gives several equivalent definitions of inaccessibility, the validity of some of the equivalences depending on the validity of the generalized continuum hypothesis.

In this note we find some further definitions or characterizations of the properties of regularity, power accessibility and accessibility of the cardinal $|X|$ in terms of the properties of certain sets of mappings of $X$ into itself and of associated mappings of $Y^{X}$ into $Y^{X}$. Of finite cardinals, 0,1 and 2 are the only regular and 0,2 are the only power inaccessible cardinals. We leave these out of account and in what follows restrict ourselves to infinite cardinals. A statement, without proof, of one of the present results (the equivalence of (1) and (4) in Theorem 1, below) occurred in the final paragraph of the author's paper [3].

1. The sets of mappings. Let $X$ be any infinite set and let $\theta: X \rightarrow X$ be a single-valued mapping of $X$ into $X$. We are interested in the following four possible properties of such a $\theta$ :

(1) $|X \theta|=|X|$;

(2) $\left|x \theta^{-1}\right|<|X|$, for all $x$ in $X$;

(3) $|X| X \theta|<| X \mid$;

(4) $\theta$ is one-to-one.

We write operators on the right and here $x \theta^{-1}$ denotes the set, possibly empty, of all elements of $X$ mapped onto $x$ by $\theta$. For any $Y, X \backslash Y$ denotes the set of elements in $X$ but not in $Y$.

Denote by $\mathscr{T}_{i}(X)$ the set of all mappings $\theta: X \rightarrow X$ satisfying property $(i)$ above, by $\mathscr{T}_{i j}(X)$ the set of all $\theta$ satisfying both properties $(i)$ and $(j)$, etc. Observe that all these sets are non-empty, for each contains all permutations of $X$. Finally, denote by $\mathscr{T}(X)$ the semigroup of all mappings of $X$ into $X$, the operation of the semigroup being composition or iteration of mappings. 
We note first some obvious properties of the $\mathscr{T}_{i}(X)$. The axiom of choice will be used, without comment, wherever necessary.

$\mathscr{T}_{4}(X)$ is clearly a subsemigroup of $\mathscr{T}(X)$ and further is contained in $\mathscr{T}_{1}(X)$ and $\mathscr{T}_{2}(X)$. Not quite so obvious is the fact that $\mathscr{T}_{3}(X)$ is a subsemigroup of $\mathscr{T}(X)$. To see this consider $\theta, \phi$ in $\mathscr{T}_{3}(X)$. Then, since

$$
X \phi \backslash X \theta \phi \subseteq(X \backslash X \theta) \phi
$$

and $|X| X \theta|<| X \mid$, therefore $|X \theta \backslash X \theta \phi|<|X|$. Hence, since

$$
X \backslash X \theta \phi=(X \backslash X \phi) \cup(X \phi \backslash X \theta \phi)
$$

and $|X \backslash X \phi|<|X|$, it follows that $|X \backslash X \theta \phi|<|X|$. Thus $\theta \phi \in \mathscr{T}_{3}(X)$, which proves that $\mathscr{T}_{3}(X)$ is a subsemigroup of $\mathscr{T}(X) . \mathscr{T}_{3}(X)$ is further a subset of $\mathscr{T}_{1}(X)$. For if $|X| X \theta \mid$ $<|X|$, it necessarily follows that $|X \theta|=|X| \cdot \mathscr{T}_{1}(X)$ is easily seen to be never a semigroup and, as we shall see below, $\mathscr{T}_{2}(X)$ is not necessarily a semigroup, nor is there necessarily a containment relation between $\mathscr{T}_{1}(X)$ and $\mathscr{T}_{2}(X)$. There is never a containment relation between $\mathscr{T}_{3}(X)$ and $\mathscr{T}_{4}(X)$ nor between $\mathscr{T}_{2}(X)$ and $\mathscr{T}_{3}(X)$.

Now let $Y$ be any set of cardinal greater than one. Any element $\theta$ of $\mathscr{T}(X)$ determines an element $\theta^{*}$ of $\mathscr{T}\left(Y^{x}\right)$ defined thus:

$$
x\left(f \theta^{*}\right)=(x \theta) f \quad\left(x \in X, f \in Y^{X}\right) .
$$

Denote the set of all such $\theta^{*}$ determined by $\theta$ in $\mathscr{T}(X)$ by $\mathscr{T}^{*}(X) . \mathscr{T}^{*}(X)$ depends, of course, also on $Y$, and we shall write $\mathscr{T}^{*}(X, Y)$ when we wish to make this dependence clear.

It is easy to verify that $(\theta \phi)^{*}=\phi^{*} \theta^{*}$, so that $\theta \rightarrow \theta^{*}$ is an antihomomorphism of $\mathscr{T}(X)$ onto $\mathscr{T}^{*}(X)$. Suppose that $\theta^{*}=\phi^{*}$ and that $\theta \neq \phi$. Then there exists $x$ in $X$ such that $x \theta=x_{1}, x \phi=x_{2}$ and $x_{1} \neq x_{2}$. Since $|Y|>1$, we can choose $y_{1}, y_{2}\left(y_{1} \neq y_{2}\right)$ in $Y$ and an element $f$ of $Y^{X}$ such that $x_{1} f=y_{1}$ and $x_{2} f=y_{2}$. Thus $x\left(f \theta^{*}\right)=(x \theta) f=x_{1} f=y_{1}$, while $x\left(f \phi^{*}\right)=(x \phi) f=x_{2} f=y_{2}$. Thus $f \theta^{*} \neq f \phi^{*}$, and so $\theta^{*} \neq \phi^{*}$, contrary to assumption. Consequently the mapping $\theta \rightarrow \theta^{*}$ is one-to-one and so is an anti-isomorphism of $\mathscr{T}(X)$ onto $\mathscr{T}^{*}(X)$.

In general if $\mathscr{S}$ is any subset of $\mathscr{T}(X)$ we shall denote by $\mathscr{S}^{*}$ the set of $\theta^{*}$ such that $\theta \in \mathscr{S}$. It follows from the above remarks that, if $|Y|>1, \mathscr{S}^{*}$ is a subsemigroup of $\mathscr{T}\left(Y^{X}\right)$ if and only if $\mathscr{S}$ is a subsemigroup of $\mathscr{T}(X)$.

2. Regular cardinals. We now use the sets described in the previous section to obtain characterization of regular, power inaccessible and inaccessible cardinals.

THEOREM 1. The following assertions about an infinite set $X$ are equivalent :

(1) $|X|$ is regular.

(2) $\mathscr{T}_{2}(X) \subseteq \mathscr{T}_{1}(X)$.

(3) $\mathscr{T}_{2}(X)$ is a subsemigroup of $\mathscr{T}(X)$.

(4) $\mathscr{T}_{12}(X)$ is a subsemigroup of $\mathscr{T}(X)$.

(5) $\mathscr{T}_{23}(X)$ is a subsemigroup of $\mathscr{T}(X)$. 
Proof. The equivalence of (1) and (2) follows directly from the definition of regularity. For suppose that $|X|$ is regular, and let $\theta \in \mathscr{T}_{2}(X)$. Should $|X \theta| \neq|X|$, then $|X \theta|<|X|$, and $\left\{y \theta^{-1}: y \in X \theta\right\}$ is a cover of $X$ by a set, of cardinal less than $|X|$, of sets each of cardinal less than $|X|$. This conflicts with the regularity of $|X|$. Consequently (1) implies (2). Conversely, suppose that (2) holds and that $|X|$ is not regular. Then there exists a disjoint cover $\left\{X_{i}: i \in I\right\}$ of $X$ such that $\left|X_{i}\right|<|X|$, for each $i$ in $I$, and $|I|<|X|$. Let $\theta$ be a mapping which, for each $i$, maps $X_{i}$ onto $x_{i}$, where $x_{i} \in X_{i}$. Then we easily have that $\theta \in \mathscr{T}_{2}(X)$. However, clearly $|X \theta|=|I|$, which, is less than $|X|$, by assumption. Thus $\theta \notin \mathscr{T}_{1}(X)$, which conflicts with the assumption that (2) holds. Thus $|X|$ must be regular. Hence (2) implies (1). Thus (1) and (2) are equivalent.

Suppose again that $|X|$ is regular. Let $\theta, \phi \in \mathscr{T}_{2}(X)$. Let $X \theta \phi=Y$. Let $y \in Y$ and consider the set $y(\theta \phi)^{-1}=\left(y \phi^{-1}\right) \theta^{-1}$. Let $y \phi^{-1}=Z$; so that, since $\phi \in \mathscr{T}_{2}(X),|Z|<|X|$. Then

$$
y(\theta \phi)^{-1}=\bigcup\left\{z \theta^{-1}: z \in Z\right\} .
$$

Because $\theta \in \mathscr{T}_{2}(X)$, each $\left|z \theta^{-1}\right|<|X|$. Hence, because $|X|$ is regular, $\left|y(\theta \phi)^{-1}\right|<|X|$. Thus $\theta \phi \in \mathscr{T}_{2}(X)$. Consequently $\mathscr{T}_{2}(X)$ is a semigroup, and we have shown that (1) implies (3).

That (1) also implies (4) and (5) now follows easily (recall that $\mathscr{T}_{3}(X)$ is a semigroup).

To prove conversely that each of (3), (4) and (5) imply (1) it will suffice to show that, when $|X|$ is not regular, then we can choose $\theta$ in $\mathscr{T}_{123}(X)$ such that $\theta^{2} \notin \mathscr{T}_{2}(X)$. For then $\theta$ belongs to each of the sets $\mathscr{T}_{2}(X), \mathscr{T}_{12}(X)$ and $\mathscr{T}_{23}(X), Y_{12}(X)$ while $\theta^{2}$ belongs to none of them.

Suppose then that $|X|$ is not regular, so that

$$
X=\bigcup\left\{X_{i}: i \in I\right\},
$$

where $|I|<|X|$ and $\left|X_{i}\right|<|X|$ for each $i$ in $I$, for some disjoint cover $\left\{X_{i}\right\}$. There will be no loss of generality in supposing that $I \subseteq X$. Let $Y=X \mid I$. Since $X$ is infinite, $|Y|=|X|$ and we may further suppose that $Y=B \cup C$, where $B$ and $C$ are disjoint, that $|B|=|C|=|Y|=|X|$ and that $B$ and $C$ have disjoint covers

$$
\begin{aligned}
& B=\bigcup\left\{B_{i}: i \in I\right\}, \\
& C=\bigcup\left\{C_{i}: i \in I\right\},
\end{aligned}
$$

with $\left|B_{l}\right|=\left|C_{l}\right|=\left|X_{i}\right|$, for each $i$ in $I$. Now define $\theta: X \rightarrow Z$, as follows:

$$
\theta: I \rightarrow j \quad \text { (for some fixed } j \text { in } I \text { ); }
$$

if $\left|X_{l}\right|$ is infinite, define

if $\left|X_{l}\right|$ is finite, define

$$
\begin{aligned}
& \theta: B_{i} \rightarrow i \\
& \theta: C_{i} \rightarrow B_{i} \cup C_{i}, \quad \text { one-to-one and onto; }
\end{aligned}
$$

Then

$$
\theta: B_{l} \cup C_{i} \rightarrow B_{i} \cup C_{i} \text {, the identity mapping. }
$$

$$
X \theta \supseteq \bigcup\left\{B_{i} \cup C_{i}: i \in I\right\},
$$


and consequently $|X \theta|=|X|$, so that $\theta \in \mathscr{T}_{1}(X)$. Further, $X \backslash X \theta \subseteq I$; thus $|X| X \theta|<| X \mid$ and so $\theta \in \mathscr{T}_{3}(X)$. Finally, $\left|x \theta^{-1}\right|$ is clearly always less than $|X|$. Hence $\theta \in \mathscr{T}_{2}(X)$. Thus we have shown that $\theta \in \mathscr{T}_{123}(X)$.

Consider now the set

$$
D=\bigcup\left\{B_{i}:\left|X_{i}\right| \text { is finite, } i \in I\right\} .
$$

This set is either finite or of cardinal less than or equal to $|I|$. In either case, since $|I|<|X|,|X|$ is infinite, and

it follows that

$$
\left|\cup\left\{B_{i}: i \in I\right\}\right|=|B|=|X| \text {, }
$$

Now

$$
|E|=|X| \text {, where } E=B \mid D \text {. }
$$

$$
j\left(\theta^{2}\right)^{-1} \supseteq I \theta^{-1} \supseteq E .
$$

Consequently, $\left|j\left(\theta^{2}\right)^{-1}\right|=|X|$, and so $\theta^{2} \notin \mathscr{T}_{2}(X)$. This completes the proof of the theorem.

The results of Theorem 1 can be amplified in various ways. We need the extensions given in the following lemma for our treatment of power inaccessible and inaccessible cardinals in the next section.

Let $2(Y)$ denote the condition on $\theta$ (depending on a given set $Y$ for which $|Y| \leqq|X|$ ),

$$
2(Y):\left|x \theta^{-1}\right|<|Y| \leqq|X| ;
$$

and let $\mathscr{T}_{2(Y)}(X)$ denote the set of all elements of $\mathscr{T}(X)$ which satisfy condition $2(Y)$. For the conjunction of conditions we use the same notation as before. An argument similar to that used to prove Theorem 1 enables us to prove the

Lemma. Let $Y$ be an infinite set and $X$ a set such that $|Y| \leqq|X|$. Then the following assertions about $|Y|$ are equivalent:

(1) $|Y|$ is regular.

(2) $\mathscr{T}_{2(Y)}(X)$ is a subsemigroup of $\mathscr{T}(X)$.

(3) $\mathscr{T}_{2(Y) 3}(X)$ is a subsemigroup of $\mathscr{T}(X)$.

3. Power inaccessible and inaccessible cardinals. In the next theorem we obtain characterizations of power inaccessible cardinals.

THEOREM 2. The following assertions about an infinite set $X$ are equivalent:

(1) $|X|$ is power inaccessible.

(2) $\mathscr{T}^{*}(X, Y) \subseteq \mathscr{T}_{2(X)}\left(Y^{X}\right)$, for all $Y$ such that $1<|Y|<|X|$.

(3) $\mathscr{T}^{*}{ }_{23}(X, Y) \subseteq \mathscr{T}_{2(X)}\left(Y^{X}\right)$, for all $Y$ such that $1<|Y|<|X|$.

(4) $\mathscr{T}^{*}{ }_{34}(X, Y) \subseteq \mathscr{T}_{2(X)}\left(Y^{X}\right)$, for all $Y$ such that $1<|Y|<|X|$.

Proof. Let $|X|$ be power inaccessible and consider $\theta^{*}$ in $\mathscr{T}^{*}{ }_{3}(X, Y)$, where $\theta \in \mathscr{T}_{3}(X)$ (see $\S 1$ ). From the definition of $\mathscr{T}_{3}(X)$, it follows that $Z=X \backslash X \theta$ is of cardinal less than 
$|X|$. Let $f, g \in Y^{x}$. From the definition of $\theta^{*}$ it follows that $f \theta^{*}=g \theta^{*}$ if and only if $(x \theta) f=(x \theta) g$ for all $x$ in $X$, i.e. if and only if $f$ and $g$ agree on $X \theta$. Consequently, when $f$ is given, a mapping $g$ such that $f \theta^{*}=g \theta^{*}$ may be defined arbitrarily on $Z$. It follows that, for any $f$ in $Y^{X}, f \theta^{*-1}$ is either empty or of cardinal $\left|Y^{z}\right|$. Consequently, since $|X|$ is power inaccessible, $\theta^{*} \in \mathscr{T}_{2(X)}\left(Y^{X}\right)$ when $|Y|<|X|$. Hence (1) implies (2).

Since $\mathscr{T}^{*}{ }_{34}(X, Y)$ and $\mathscr{T}^{*}{ }_{23}(X, Y)$ are subsets of $\mathscr{T}^{*}(X, Y)$, we have immediately that (1) also implies (3) and (4).

To prove the converse implications, suppose that $|X|$ is not power inaccessible, so that there exist $Y$ and $Z$, with $1<|Y|<|X|$ and $|Z|<|X|$, such that $\left|Y^{Z}\right|=|X|$. We can assume that $Z \subseteq X$, when, since $|Z|<|X|$, we have $|X| Z|=| X \mid$. Now let $\theta$ be any $(1,1)$-mapping of $X$ onto $X \mid Z$. Then we easily see that $\theta \in \mathscr{T}_{234}(X)$. However, for any $f$ in $Y^{x} \theta^{*},\left|f \theta^{*-1}\right|=\left|Y^{Z}\right|=|X|$. Thus $\theta^{*} \notin \mathscr{T}_{2(X)}\left(Y^{X}\right)$. That (2), (3) and (4) do not hold for $X$ now follows immediately.

As an immediate corollary to Theorem 2, the lemma, and the remark at the end of $\$ 1$, we have the following characterization of inaccessible cardinals.

THEOREM 3. Let $X$ be an infinite set. Then $|X|$ is inaccessible if and only if, for all $Y$ such that $1<|Y|<|X|, \mathscr{T}^{*}{ }_{23}(X, Y)$ is a subsemigroup of $\mathscr{T}\left(Y^{X}\right)$ contained in $\mathscr{T}_{2(X)}\left(Y^{X}\right)$.

\section{REFERENCES}

1. H. Bachmann, Transfinite Zahlen (Ergebnisse der Math. N.F. 1, Springer, 1955).

2. A. Tarski, Über unerreichbare Kardinalzahlen, Fund. Math. 30 (1938), 68-89.

3. G. B. Preston, Embedding any semigroup in a $\mathscr{D}$-simple semigroup, Trans. Amer. Math. Soc. 93 (1959), 351-355.

Royal Military College of Science

SHRIVENHAM 